\title{
Fast magnetohydrodynamic oscillations in an elliptical coronal arcade
}

\begin{abstract}
A. J. Díaz
Mathematical Institute, University of St Andrews, St Andrews KY16 9SS, Scotland, UK

e-mail: antonio@mcs.st-and.ac.uk

Received 10 March 2006 / Accepted 10 June 2006

ABSTRACT

Aims. A model of a elliptically shaped coronal arcade with piecewise constant density is discussed to explore the effects of curvature on radially polarised fast modes. It is important to test whether the main results in the straight and cylindrical geometries can be extrapolated to these more complex equilibria.

Methods. An equilibrium model for a force-free, line-tied elliptical arcade is introduced and a partial differential equation is derived for the velocity perturbation of the fast modes, which is solved analytically. The properties of the modes are studied in terms of the dispersion relation, which depends on the eccentricity, the arcade width, and the density contrast.

Results. Modes mainly contained in the cavity below the arcade are also present, and have avoided crossings with the modes of the arcade. Even the fundamental mode becomes leaky due to curvature. Approximated relations are deduced for the frequency of the modes and the spatial structure is discussed, focusing on the different families through which a rich mode spectrum can be classified. Conclusions. The different types of modes of the spectrum are described and its relevance to observations is discussed. The periods obtained in Cartesian geometry provide a reasonable approximation, but this geometry lacks some other key ingredients: the damping rates are different and some types of modes present in the elliptical geometry are not sustained in the straight slab.
\end{abstract}

Key words. Sun: oscillations - Sun: magnetic fields - Sun: corona

\section{Introduction}

Observations show clearly that the solar corona is structured in regions with open magnetic fields or coronal holes and in regions with closed magnetic field (with field endpoints anchored in the photosphere). Closed regions are composed of myriads of coronal loops, sometimes aligned together forming arcades with a wide range of densities and temperatures. Oscillations are observed in these coronal structures, especially in loops (see the reviews in Aschwanden 2004; and Nakariakov \& Verwichte 2005) and also in coronal arcades (Verwichte et al. 2004, 2005)

The oscillatory properties of an isolated, infinite, uniform, Cartesian slab were discussed by Edwin \& Roberts (1982), and its cylindrically-symmetric flux counterpart by Edwin \& Roberts (1983) and Roberts et al. (1984). These works provide a basic theory for understanding the oscillations of coronal loops and arcades, and their results are used to extract information regarding the modes (coronal seismology). In particular, certain modes are known to be highly dispersive and different families of modes are supported by the flux tube: kink or sausage modes, depending of the azimuthal wavenumber, or body and surface modes, depending on the radial wavenumber, may arise. However, it is not known how these relatively simple models are affected by the complexities of the real corona. Most theoretical studies of coronal oscillations tend to study straight field configurations, exploring a variety of aspects; for example, the effect of structure along the axis (Roberts et al. 1984; Díaz et al. 2004; Van Doorsselaere et al. 2004; Andries et al. 2005a, 2005b; Donnelly et al. 2006; McEwan et al. 2006).

The role of curvature has been investigated during recent years. Oliver et al. (1993) studied analytically the oscillatory modes of a potential arcade. This work was extended numerically by considering non-potential and sheared arcades (Oliver et al. 1996; Arregui et al. 2001, 2003, 2004a,b). Recently, the modes of a cylindrical arcade have been explored, focusing on the differences with the straight models (Smith et al. 1997; Brady $\&$ Arber 2005; Selwa et al. 2005; Verwichte et al. 2006a,b; Díaz et al. 2006). All these works show that leakage seems to be an important effect resulting from curvature, but also other interesting effects are present, such as the appearance of new families of modes or the presence of avoided crossings. However, a cylindrically symmetric arcade cannot explore the whole effects that are expected, since the curvature is a fixed parameter. To study further the role of curvature, we consider here a simple model of a potential field in cylindrical elliptical coordinates, with a density that varies across the field lines. The density variation does not model the actual stratification of the corona, but is simply a consequence of the field line divergence. Moreover, the field lines are diverging away from the photospheric footpoints, so the arcade is wider in the apex. It has been shown numerically that field divergence is important for mode coupling (Beliën et al. 1997; Bogdan et al. 2003; Hasan et al. 2005), but in our analysis we can decouple the modes and focus on the fast modes. Finally, the results of the elliptical arcade are compared with those of straight Cartesian and cylindrical geometries.

\section{Equilibrium model and wave equations}

The first task is to find a suitable equilibrium for our analysis. The arcade is modelled as a curved structure with a density enhancement over the embedding coronal plasma (see Fig. 1). 


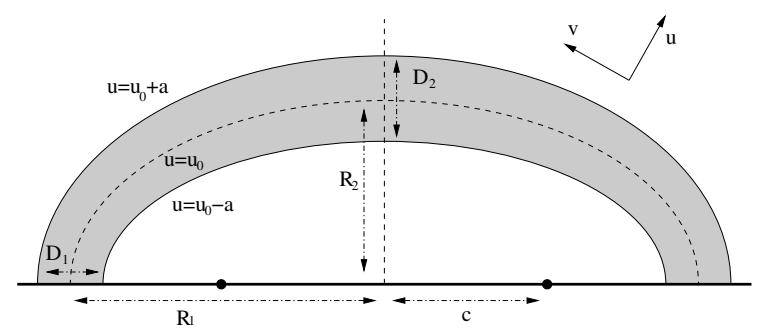

Fig. 1. Sketch of the equilibrium configuration representing an elliptical magnetic arcade. The dense zone between $u=u_{0}-a$ and $u=u_{0}+a$ has been shaded in grey. The magnetic field $\boldsymbol{B}_{0}$ points in the $v$-direction. The configuration is described by cylindrical elliptical coordinates $(u, v, z)$, with invariance in the $z$-direction.

\subsection{Cylindrical elliptic coordinate system}

Throughout this paper we use cylindrical elliptic coordinates $(u, v, z)$, which are related with the Cartesian system by the relations (Spiegel 1968)

$x=c \cosh u \cos v$,

$y=c \sinh u \sin v$.

In this orthogonal coordinate system the scale factors are the same for the two coordinates $(u, v)$, namely

$h^{2}=c^{2}\left(\sinh ^{2} u+\sin ^{2} v\right)=c^{2}(\cosh 2 u-\cos 2 v) / 2$.

The domain is restricted to the region $0 \leq u<\infty$ and $0 \leq v \leq \pi$, with $v=0$ and $v=\pi$ marking the position of the dense photosphere.

The curves $u=u_{0}$ constant are ellipses which satisfy

$\left(\frac{x}{c \cosh u_{0}}\right)^{2}+\left(\frac{y}{c \sinh u_{0}}\right)^{2}=1$,

and the curves with $v=v_{0}$ are hyperbolae which satisfy

$\left(\frac{x}{c \cos v_{0}}\right)^{2}-\left(\frac{y}{c \sin v_{0}}\right)^{2}=1$.

Notice from Eqs. (3) and (4) that the parameter $c$ in Eqs. (1) marks the foci of the different curves. The eccentricity of the ellipses in Eq. (3) is

$\varepsilon=1 / \cosh u_{0}$.

For small values of the coordinate $u$ the curves are very eccentric ellipses (and in the limit $u_{0} \rightarrow 0$ they become a straight line between the two foci); for large values the eccentricity is very low and in the limit $u_{0} \rightarrow \infty$ they become circles of radius

$R \approx c \cosh u_{0} \approx c \sinh u_{0} \approx c \mathrm{e}^{u_{0}} / 2$.

The coordinate $u$ plays a similar role to the cylindrical coordinate $r$. The scale factors in Eq. (2) are in this limit

$h^{2} \approx c^{2} \sinh ^{2} u_{0} \approx R^{2}$.

\subsection{Plasma equilibrium configuration}

Our equilibrium consists of a dense coronal loop embedded in a coronal medium (Fig. 1). The whole configuration is invariant in the $z$-direction. The dense material lies between the ellipses $u=u_{0} \pm a$, with $u=u_{0}$ being the central curve of the slab axis. The equilibrium density is then:

$\rho_{0}(u, v)= \begin{cases}\rho_{1}\left(h / R_{1}\right)^{\alpha}, & \left|u-u_{0}\right| \leq a, \\ \rho_{\mathrm{c}}\left(h / R_{1}\right)^{\alpha}, & \left|u-u_{0}\right|>a .\end{cases}$
Hereafter the value $\alpha=-4$ is fixed, but other choices are possible. $R_{1}$ is the loop half-distance between the two footpoints, which will be discussed in detail later. We could have chosen the arcade length $L$ for the scaling of the density profile, but we choose $R_{1}$ to be consistent with the cylindrical model equilibrium profile in Verwichte et al. (2006a).

There are two important parameters regarding the size, namely the distance between the footpoints, $2 R_{1}$, and the height of the summit $R_{2}$, with

$R_{1}=c \cosh u_{0}, \quad R_{2}=c \sinh u_{0}$.

As $u_{0}$ is increased $R_{1}$ and $R_{2}$ become similar and can be identified as the radius of the arcade. Notice that the arcade height is smaller that the half-separation between footpoints $\left(R_{2} \leq R_{1}\right)$. Similarly, there are two widths of the loop, one in the footpoints, $D_{1}$, and one in the summit, $D_{2}$, with

$D_{1}=2 c \sinh u_{0} \sinh a, \quad D_{2}=2 c \cosh u_{0} \sinh a$.

The width in the summit is bigger than in the footpoints $\left(D_{2} \geq\right.$ $D_{1}$ ), which is in accordance with theoretical and computational equilibrium models. Notice that the parameter $a$ plays a similar role to the width of the arcade, but the position of the axis $u_{0}$ is also relevant.

Finally, the length of the structure must be defined, specially for comparing with the results of the cylindrical and straight arcades. The length of the arcade is defined as the length $L$ of the magnetic field line with $u=u_{0}$,

$$
\begin{aligned}
L & =\int_{0}^{\pi} \sqrt{\left(\frac{\mathrm{d} x}{\mathrm{~d} v}\right)^{2}+\left(\frac{\mathrm{d} y}{\mathrm{~d} v}\right)^{2}} \mathrm{~d} v \\
& =2\left(c \cosh u_{0}\right) \int_{0}^{\frac{\pi}{2}} \sqrt{1-\varepsilon^{2} \cos ^{2} v} \mathrm{~d} v \\
& =2\left(c \cosh u_{0}\right) E(\varepsilon, \pi / 2) .
\end{aligned}
$$

The resulting integral $E(\varepsilon, \pi / 2)$ is a complete elliptical integral of the second kind (Abramowitz \& Stegun 1967), which can be expanded in the series

$E(\varepsilon, \pi / 2)=\frac{\pi}{2} \sum_{n=0}^{\infty} \frac{1}{n-1}\left(\frac{(2 n) !}{\left(2^{n} n !\right)^{2}}\right)^{2} \varepsilon^{2 n}$

Therefore, for small values of $u_{0}$ the eccentricity is large and the integral is close to 1 , so the length is $L \approx 2 c$; on the other hand, for large values of $u_{0}$ the integral is close to $\pi / 2$, and the length is $L \approx \pi R$ (with $R$ defined in Eq. (6)). A sketch of $E(\varepsilon, \pi / 2)$ is provided in Fig. 2.

\subsection{Equilibrium magnetic field}

We wish to construct an equilibrium magnetic field which satisfies the solenoidal and the force-free conditions and is oriented in the $v$-direction in this geometry,

$\boldsymbol{B}_{0}=B_{v}(u, v) \boldsymbol{e}_{v}$.

The condition $\nabla \cdot \boldsymbol{B}_{0}=0$ implies

$\nabla \cdot \boldsymbol{B}_{0}=\frac{\partial\left(h B_{v}\right)}{\partial v}=0$

and from the force-free condition $\nabla \times \boldsymbol{B}_{0}=0$,

$\nabla \times \boldsymbol{B}_{0}=\frac{1}{h^{2}} \frac{\partial\left(h B_{v}\right)}{\partial u} \boldsymbol{e}_{z}=0$. 


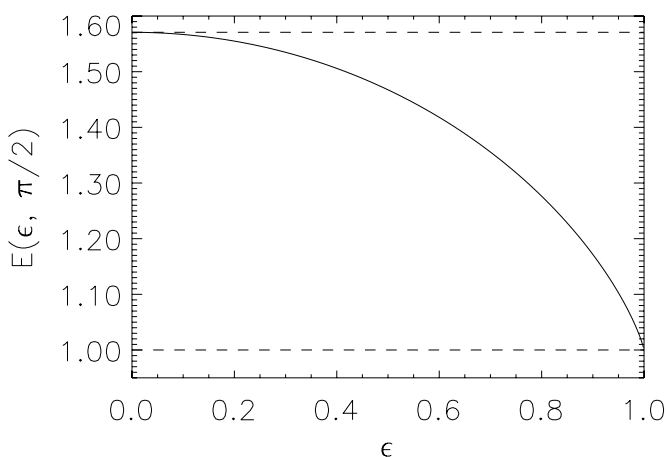

Fig. 2. Plot of $E(\varepsilon, \pi / 2)$, the complete elliptical integral of the second kind (see Eq. (11)). Two horizontal dashed lines have been overplotted marking the limiting values for $\varepsilon=0$ and $\varepsilon=1$; since $E(\varepsilon \rightarrow 0, \pi / 2) \rightarrow$ $\pi / 2$ and $E(\varepsilon \rightarrow 1, \pi / 2) \rightarrow 1$.

Therefore, the only choice is to have a dependence in the form $h B_{v}=$ const., so the only component of our equilibrium magnetic field $B_{v}$ is in the form

$B_{v}(u, v)=\frac{B_{0} R_{1}}{h}=\frac{B_{0}}{\left(\sinh ^{2} u+\sin ^{2} v\right)^{1 / 2}} \frac{R_{1}}{c}$.

Surface plots of $B_{v}$ and $c_{\mathrm{A}}$ are shown in Fig. 3. This equilibrium configuration is produced by two line currents situated in the foci of the ellipses, so the magnetic field is diverging in these two points (in a similar way to the cylindrical case in $r=0$ ). Again, the scaling distance has been chosen to allow comparison with the cylindrical models.

\subsection{Wave equations}

The starting point for our MHD wave analysis are the linearised ideal MHD equations for zero- $\beta$,

$\rho_{0}(u, v) \frac{\partial \boldsymbol{v}}{\partial t}=\frac{1}{\mu}(\nabla \times \boldsymbol{B}) \times \boldsymbol{B}_{0}+\frac{1}{\mu}\left(\nabla \times \boldsymbol{B}_{0}\right) \times \boldsymbol{B}$,

$\frac{\partial \boldsymbol{B}}{\partial t}=\nabla \times\left(\boldsymbol{v} \times \boldsymbol{B}_{0}\right)$,

$\nabla \cdot \boldsymbol{B}=0$

These equations describe the behaviour of the perturbed magnetic field $\boldsymbol{B}$, velocity $\boldsymbol{v}$, from which the perturbed density $\rho$ and pressure $p$ can be obtained. For zero- $\beta$ plasma all oscillations are purely a response to magnetic forces; in a uniform medium, these modes would be the Alfvén and fast magnetoacoustic waves.

The equation of motion (Eq. (17)) leads to

$\mu \rho_{0}(u, v) \frac{\partial^{2} \boldsymbol{v}}{\partial t^{2}}=-\boldsymbol{B}_{0} \times\left(\nabla \times \frac{\partial \boldsymbol{B}}{\partial t}\right)$,

in which Eq. (15) has been applied. Using the induction equation (Eq. (18)) and the invariance along the $z$-coordinate (so derivatives with respect to $z$ are zero), it follows that

$\frac{\partial \boldsymbol{B}}{\partial t}=\boldsymbol{e}_{u} \frac{1}{h} \frac{\partial}{\partial v}\left(v_{u} B_{v}\right)-\boldsymbol{e}_{v} \frac{1}{h} \frac{\partial}{\partial u}\left(v_{u} B_{v}\right)+\boldsymbol{e}_{z} \frac{B_{v}}{h} \frac{\partial v_{z}}{\partial v}$.

Substituting this result into Eq. (20) gives after some algebra the following differential equations for the components of the perturbed velocity $\boldsymbol{v}=\left(v_{u}, v_{v}, v_{z}\right)$,

$\frac{\partial^{2} v_{u}}{\partial t^{2}}-\frac{B_{v}}{h^{2} \mu \rho_{0}}\left[\frac{\partial^{2}}{\partial u^{2}}\left(v_{u} B_{v}\right)-\frac{\partial^{2}}{\partial v^{2}}\left(v_{u} B_{v}\right)\right]=0$,

$$
\begin{aligned}
& \frac{\partial^{2} v_{v}}{\partial t^{2}}=0 \\
& \frac{\partial^{2} v_{z}}{\partial t^{2}}-\frac{B_{v}^{2}}{\mu \rho_{0}} \frac{\partial}{\partial v}\left(\frac{1}{h^{2}} \frac{\partial v_{z}}{\partial v}\right)=0
\end{aligned}
$$

Thus, $v_{v}=0$ from Eq. (23), in a similar way to the azimuthal velocity being identically zero in the cylindrical geometry. Equation (24) represents an Alfvén wave polarised in the $z$-direction, with each field line oscillating at its own local Alfvén frequency, forming a continuous spectrum. If propagation along the $z$-axis is permitted, then the modes become strongly coupled (Beliën et al. 1997), but here we concentrate on the fast mode alone by setting $v_{z}=0$.

Equation (22) governing the fast modes can be rewritten using a new dependent variable $w=v_{u} / h$ and the expression of the equilibrium magnetic field, Eq. (16), as

$\frac{\partial^{2} w}{\partial u^{2}}+\frac{\partial^{2} w}{\partial v^{2}}-\frac{h^{4}}{R_{1}^{2}} \frac{\mu \rho_{0}}{B_{0}^{2}} \frac{\partial^{2} w}{\partial t^{2}}=0$

With the choice of a equilibrium density profile so that $\alpha=-4$ in Eq. (8) we obtain

$\frac{\partial^{2} w}{\partial u^{2}}+\frac{\partial^{2} w}{\partial v^{2}}-\frac{R_{1}^{2}}{c_{\mathrm{A} 0}^{2}} \frac{\partial^{2} w}{\partial t^{2}}=0$

where $c_{\mathrm{A} 0}=B_{0} / \sqrt{\mu \rho_{0}}$ is the Alfvén speed at $u=u_{0}, v=\pi / 2$ ( $x=0, y=R_{2}$, at the apex of the arcade).

The partial differential equation Eq. (26) admits separation of variables in the form

$w(u, v, t)=f(u) g(v) \mathrm{e}^{\mathrm{i} \omega t}$

where the time dependence has been Fourier-analysed, giving

$\frac{\mathrm{d}^{2} g}{\mathrm{~d} v^{2}}+m^{2} g=0$,
$\frac{\mathrm{d}^{2} f}{\mathrm{~d} u^{2}}+\left(\frac{\omega^{2} L^{2}}{c_{\mathrm{A} 0}^{2}} \frac{1}{4 E(\varepsilon, \pi / 2)^{2}}-m^{2}\right) f=0$,

with $m$ as a separation constant and $R_{1} / L=1 /(2 E(\varepsilon, \pi / 2))$ from Eqs. (9) and (11). The solutions of these equations are in the form of trigonometric and hyperbolic functions, respectively,

$g(v)=C_{1} \cos m v+C_{2} \sin m v$,

$f(u)=C_{3} \cosh \kappa_{j} u+C_{4} \sinh \kappa_{j} u$,

where the following definition has been used,

$\kappa_{j}^{2}=m^{2}-\frac{\omega^{2} L^{2}}{c_{\mathrm{A} j}^{2}} \frac{1}{4 E(\varepsilon, \pi / 2)^{2}}, \quad j=1, \mathrm{c}$.

\section{Dispersion relation}

The next step is to apply the solutions to our model. First, the photospheric line-tying demands that the solution must be zero at $x=0$, which is equivalent to

$v_{u}(u, v=0, t)=v_{u}(u, v=\pi, t)=v_{u}(u=0, v, t)=0$.

The first and second condition demand that $C_{1}=0$ and that the separation constant $m$ must be an positive integer, $m=1,2, \ldots$. Hence, we find also the same different types of families that are present in the cylindrical geometry. 

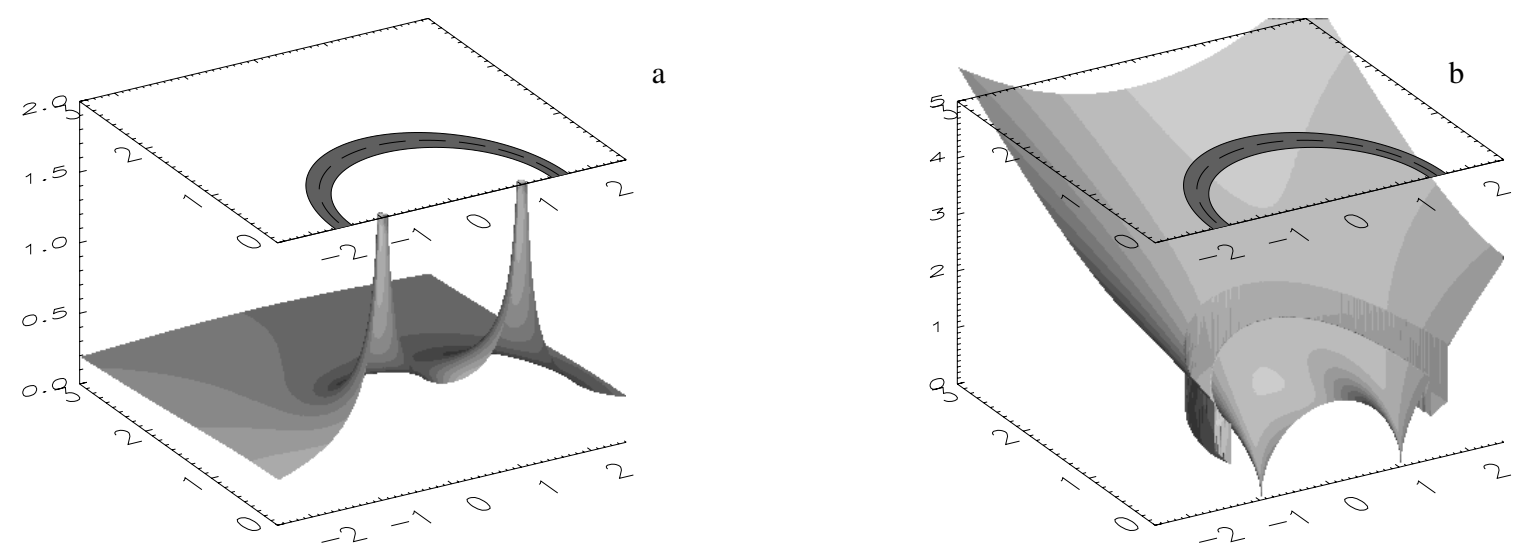

Fig. 3. Surface plots of the $B_{v}$ component of the equilibrium magnetic field (panel a)) and the Alfvén speed (panel b)). Sketches of the arcade have been overplotted in each upper plane.

The general solution has three different regions: a dense arcade plasma in $u_{0}-a<u \leq u_{0}+a$, and coronal plasma in the surrounding regions. Then

$w(u, v)=\sin m v \begin{cases}A_{1} \sinh \kappa_{\mathrm{c}} u, & u \leq u_{0}-a, \\ A_{2} \sinh \kappa_{1} u+A_{3} \cosh \kappa_{1} u, & \left|u_{0}-u\right| \leq a, \\ A_{4} \mathrm{e}^{\kappa_{\mathrm{c}}\left(u-u_{0}-a\right)}, & u>u_{0}+a,\end{cases}$

with $\kappa_{1}$ and $\kappa_{\mathrm{c}}$ defined by using Eq. (31) with the suitable Alfvén speed. We consider such solutions in conjunction with the boundary conditions

$\left[v_{u}\right]=\left[\frac{\partial v_{u}}{\partial u}\right]=0$.

These boundary conditions may be deduced from first principles (Goedbloed 1983), much as in Díaz (2004).

The form of the solution in the outer region in Eq. (33), $u>u_{0}+a$, has been selected so that it decays as $u \rightarrow \infty$. Because the Alfvén speed increases as we go far from the arcade, trapped waves are here possible, as predicted in the cylindrical model in Verwichte et al. (2006a), since the equilibrium density dependence has been chosen so in the limit of low eccentricity is equivalent to theirs). In the inner region, $u \leq u_{0}-a$, the solution in Eq. (33) has been chosen to satisfy the last condition in Eq. (32).

Applying the boundary conditions (Eq. (34)) the constant coefficients can be related. The following dispersion relation is obtained

$-\frac{\kappa_{\mathrm{c}}}{\kappa_{1}} \tanh \left\{2 a \kappa_{1}\right\}=\frac{1+\tanh \left\{\kappa_{\mathrm{c}}\left(u_{0}-a\right)\right\}}{1+\tanh \left\{\kappa_{\mathrm{c}}\left(u_{0}-a\right)\right\} \kappa_{1}^{2} / \kappa_{\mathrm{c}}^{2}}$,

which, after some algebra, can be written also as

$\mathrm{e}^{4 a \kappa_{1}}=\frac{\left(\kappa_{\mathrm{c}} \operatorname{coth}\left\{\kappa_{\mathrm{c}}\left(u_{0}-a\right)\right\}-\kappa_{1}\right) \cdot\left(\kappa_{\mathrm{c}}-\kappa_{1}\right)}{\left(\kappa_{\mathrm{c}} \operatorname{coth}\left\{\kappa_{\mathrm{c}}\left(u_{0}-a\right)\right\}+\kappa_{1}\right) \cdot\left(\kappa_{\mathrm{c}}+\kappa_{1}\right)}$.

We obtain a single dispersion relation for all the modes, and there is no distinction between kink and sausage modes, contrary to the dispersion relations in cylindrical and Cartesian geometry. Moreover, both $A_{2}$ and $A_{3}$ in Eq. (33) are necessary to have solutions, pointing again to the fact that here the kink and sausage modes are not a natural description for this problem, since the two halves in which the axis $u=u_{0}$ divides the arcade are not equivalent.
There are various types of solution depending on the value of $m$. If $m=1$, then the solution has an anti-node at the arcade summit; we name this as the vertical mode to differentiate it from the transversal rocking modes (with displacement in the $z$-direction in a 3D model). Modes with $m=2$ have a node at the arcade apex and appear to shift the arcade laterally; these we term the swaying modes (see also Díaz et al. (2006)). The modes may also be classified, depending on their symmetry with respect to the arcade axis, as kink (even) and sausage (odd) modes, although this classification is not evident in Eqs. (33) and (35). Families with higher values of $m$ are less relevant for the standing modes. We discuss further these features when studying the resulting spatial distributions.

\subsection{Limit of low eccentricity}

Our first check is to test if the limit of a cylindrical arcade studied in Verwichte et al. (2006a) is recovered. This limit is achieved when the lower boundary of the arcade is far away from the loci, so $u_{0}-a \gg 1$. Under that condition Eq. (6) is satisfied, so $\tanh \left\{\kappa_{\mathrm{c}}\left(u_{0}-a\right)\right\} \approx 1$, and Eq. (36) becomes

$\mathrm{e}^{\kappa_{1}(2 a)} \mathrm{e}^{\mathrm{i} \pi n}=\frac{\left(\kappa_{\mathrm{c}}-\kappa_{1}\right)}{\left(\kappa_{\mathrm{c}}+\kappa_{1}\right)}$.

The extra factor comes from the fact that the square root has been taken. The first exponential function in the left-hand side is expressed as

$\mathrm{e}^{\kappa_{1}(2 a)}=\mathrm{e}^{\kappa_{1}\left[\left(u_{0}+a\right)-\left(u_{0}-a\right)\right]}=\mathrm{e}^{\kappa_{1}} \frac{\mathrm{e}^{u_{0}+a} c / 2}{\mathrm{e}^{u_{0}-a} c / 2}=\mathrm{e}^{\kappa_{1}} \frac{R_{+}}{R_{-}}$,

where $R_{ \pm}$are the radius of the boundaries of the arcade. Hence, Eq. (37) becomes

$\mathrm{e}^{\kappa_{1} \ln R_{+} / R_{-}} \mathrm{e}^{\mathrm{i} \pi n}=\frac{\left(\kappa_{\mathrm{c}}-\kappa_{1}\right)}{\left(\kappa_{\mathrm{c}}+\kappa_{1}\right)}$,

which is exactly Eq. (17) in Verwichte et al. (2006a). Notice also the definition of the $\kappa$ in Eq. (31) is the same definition that their Eq. (11) in this limit, since $E(\varepsilon, \pi / 2) \approx \pi / 2$ when $\varepsilon \ll 1$, so

$\kappa_{j} \approx m^{2}-\frac{\omega^{2} L^{2}}{c_{\mathrm{A} j}^{2}} \frac{1}{\pi^{2}} \approx m^{2}-\frac{\omega^{2} R^{2}}{c_{\mathrm{A} j}^{2}}$ 


\subsection{Elliptical cavity}

Following Díaz et al. (2006), the modes of a elliptical domain with a rigid boundary at $u=u_{0}-a$ are explored. The solution to Eq. (28) is

$f(u)=D_{1} \sinh \kappa_{\mathrm{c}} u$,

in which the line-tying boundary condition $f(u=0)=0$ has been applied. Next, the rigid boundary at the arcade position means that $f\left(u_{0}-a\right)=0$, so

$\kappa_{\mathrm{c}}\left(u_{0}-a\right)=\mathrm{i} n \pi, \quad n=1,2, \ldots$

This leads to an explicit expression for the frequencies using the definition in Eq. (31),

$\frac{\omega_{n} L}{c_{\mathrm{Ac}}}=2 E(\varepsilon, \pi / 2) \sqrt{m^{2}+\frac{n^{2} \pi^{2}}{\left(u_{0}-a\right)^{2}}}$.

A straightforward conclusion from Eq. (43) is that the modes of these elliptical membranes have very high frequency for arcades with high eccentricity $\left(u_{0} \ll 1\right)$. Also, if $a=u_{0}$ there is no cavity below the arcade, so the frequencies of these modes tend to infinity as $a \rightarrow u_{0}$. On the other hand, for high eccentricity all these modes tend to the same frequency $m \pi$, which is the frequency of the fundamental mode of the arcade. This is in agreement with the result in Verwichte et al. (2006a) that for a cylindrical arcade model with this equilibrium density profile there are no modes of the cavity (other than the fundamental mode of the system, in which both the arcade and the cavity oscillate together).

\subsection{Elliptical ring}

Another interesting special case is a domain with rigid boundaries at the arcade limits $u=u_{0} \pm a$, which is a good approximation when the density contrast $\rho_{1} / \rho_{\mathrm{c}}$ is high. The solution to Eq. (28) is

$f(u)=D_{2} \sinh \kappa_{1} u+D_{3} \cosh \kappa_{1} u$.

The rigid boundary conditions $f\left(u_{0} \pm a\right)=0$ imply

$\tanh \left\{\kappa_{1}\left(u_{0}-a\right)\right\}=\tanh \left\{\kappa_{1}\left(u_{0}+a\right)\right\}$.

After some algebra, the following expression is obtained:

$\frac{\omega_{n} L}{c_{\mathrm{Ac}}}=2 E(\varepsilon, \pi / 2) \frac{\rho_{\mathrm{c}}}{\rho_{\mathrm{l}}} \sqrt{m^{2}+\frac{n^{2} \pi^{2}}{4 a^{2}}}$.

These modes have high frequency for thin structures and do not depend on the eccentricity of the arcade, except via the elliptical integral.

\section{Results}

\subsection{Dispersion relations}

We consider the dependence of the frequencies on the parameters of the model: the density contrast, $\rho_{\mathrm{l}} / \rho_{\mathrm{c}}$, the width of the arcade, related to $a$ (Eq. (10)), and the eccentricity, related to the position of the footpoints $u_{0}$ by Eq. (5).

We have represented in Fig. 4 the frequencies vs. $a$ for a fixed value of the footpoints position. For this equilibrium density profile, trapped modes are allowed but, in contrast with the results in the cylindrical geometry, the cutoff frequency depends on the
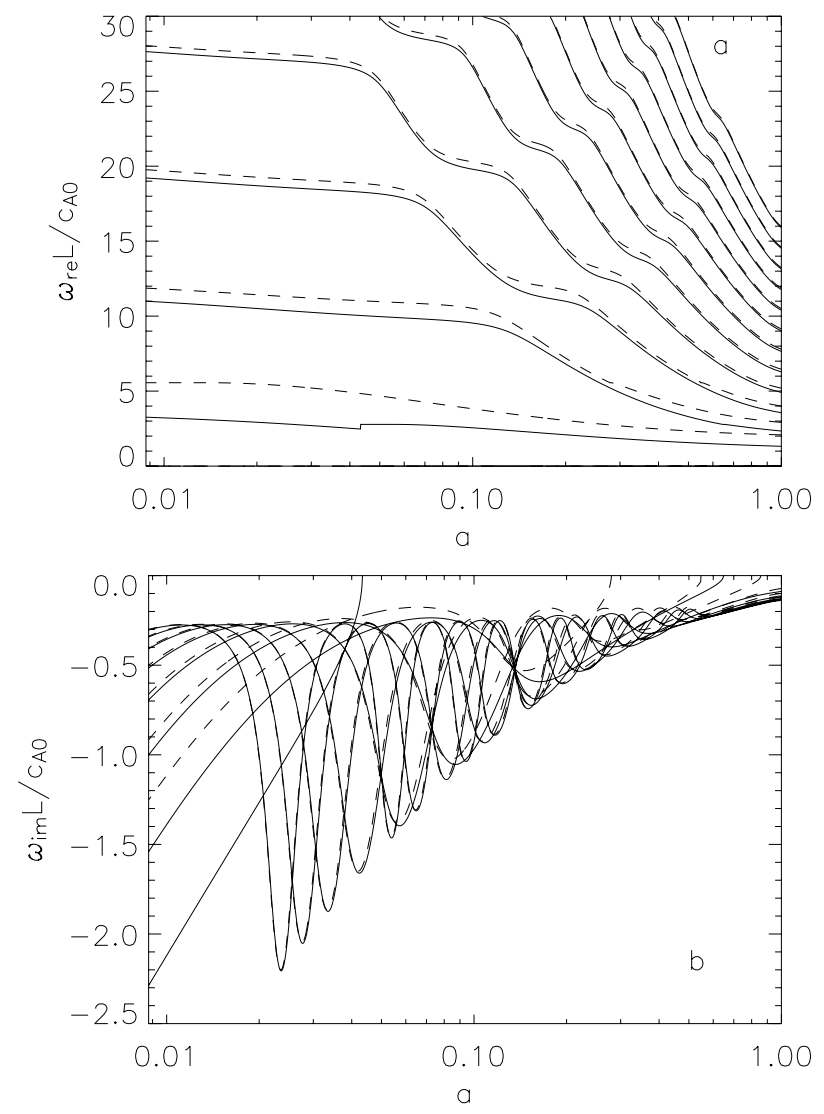

Fig. 4. Logarithmic plots of the a) real and b) imaginary parts of $\omega$ against the parameter a for vertical modes ( $m=1$, solid lines), swaying modes $(m=2$, dotted lines). The density ratio has been fixed as $\rho_{1} / \rho_{\mathrm{c}}=10$ and the footpoint parameter as $u_{0}=1$. Only the 12 lowest values in panel a) and the 7 lowest values in panel b) have been represented for each family.

eccentricity of the model, since in Eq. (31) a cutoff is achieved when $\kappa_{\mathrm{c}}=0$, or

$\frac{\omega_{\text {cutoff }} L}{c_{\mathrm{Ac}}}=2 E(\varepsilon, \pi / 2) m$.

In the limit of high eccentricity the cutoff frequency of the straight and cylindrical models, $m \pi$, is recovered. For typical values of the parameters in the solar corona just one mode in each family is in this regime (as in Fig. 4), but other modes might become trapped if the density contrast is increased, for example.

Notice the appearance of avoided crossings in Fig. 4, contrary to what it is obtained in Verwichte et al. (2006a) for a cylindrical arcade in a profile equivalent to this one. In Díaz et al. (2006) it was stated that, for a different equilibrium, these modes have spatial structure in the coronal region below the arcade, so we refer to them as cavity modes, in contrast with the arcade modes. This is due to the fact that in the elliptical geometry there are regions with low Alfvén speed below the arcade which can trap modes, and these are the modes present for low values of the arcade thickness.

Equation (43) describes quite reasonably the behaviour of the cavity modes before the avoided crossings ( $a$ small). All the families with different values of $m$ tend to have the same frequency for higher harmonics, as predicted in Eq. (43), since the term with the harmonic order $n$ becomes larger compared with the term $m$ in the square root. On the other hand, the frequency of the arcade modes is roughly inverse proportional to $a$ (Eq. (46)), a feature more apparent for higher harmonics again. 


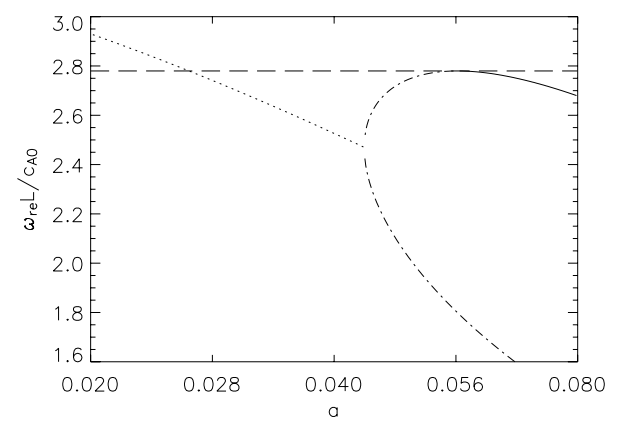

Fig. 5. Detail of Fig. 4 near the cutoff for fundamental vertical mode $(m=1)$ with the parameters $\rho_{\mathrm{l}} / \rho_{\mathrm{c}}=10$ and $u_{0}=1$. The solid line is a trapped mode and the dotted line is an outwards propagating leaky mode. Unphysical solutions have also been plotted in the dash-dotted lines corresponding to modes that increase exponentially with distance (no damping). The cutoff has been represented as a horizontal dashed line. Notice that for lower values of $a$ the leaky mode lies above the cutoff.

On the other hand, the different families of modes related to the $m$ number have similar frequencies for thick arcades, but for thin arcades the cavity modes are different. Similar conclusions are obtained if higher values of $m$ are considered. The swaying modes $(m=2)$ do not have twice the frequency of the vertical modes $(m=1)$, as happens in the Cartesian geometry.

One noticeable feature in Fig. 4 is that the even the fundamental mode has a cutoff as $a$ is lowered, but then the mode appears again below the cutoff frequency (with an imaginary part to its frequency) after a gap in the values of $a$ were no fundamental mode is present. A closer look of this region is depicted in Fig. 5 for the fundamental vertical mode. Notice that, in opposition to the straight model (Edwin \& Roberts 1982, 1983) and the cylindrical one (Verwichte et al. 2006a), the fundamental mode is no longer trapped for all the values of the arcade width, but has a cutoff and becomes leaky. On the other hand, if unphysical solutions are overplotted (as it is done in Fig. 5), we see that there is a branching point and the behaviour is completed: the previous trapped mode becomes a solution which expands exponentially away from the arcade, and this mode merges with a similar one to form a leaky mode (which is a outward propagating leaky mode, and therefore physically acceptable). These unphysical modes are also present because in Eq. (31) there is a choice of sign which leads to confined or exponentially increasing modes for real values of frequency, and to inwards or outwards propagating modes for complex values of frequency. This behaviour has been reported in the straight slab for higher harmonics (Terradas et al. 2005), and is also present in the cylindrical model (though not been discussed in Verwichte et al. 2006a). However, in the elliptical geometry even the fundamental mode has this gap and mode conversion near the cutoff. The reason is that the cutoff is lowered by the elliptical geometry (Eq. (47)), but in the limit $a \rightarrow 0$ this fundamental mode tends to a normalised frequency $m \pi$ (the same as that in the straight slab) which is above the cutoff, and hence it becomes leaky.

Next, the footpoint position is considered, which is related to the eccentricity of the arcade after rescaling with the length. The parameter $a$ is kept fixed. The real and imaginary part of the resulting frequencies have been plotted in Fig. 6 . Notice that $u_{0}$ cannot take values smaller than $a$, so the curves are stopped when $u_{0}=a$. We can see again the presence of avoided crossings between the arcade modes (lines with less inclination) and the cavity modes (there is just one for the lower value of $a$ in this figure). Equation (43) describes quite reasonably the behaviour of the
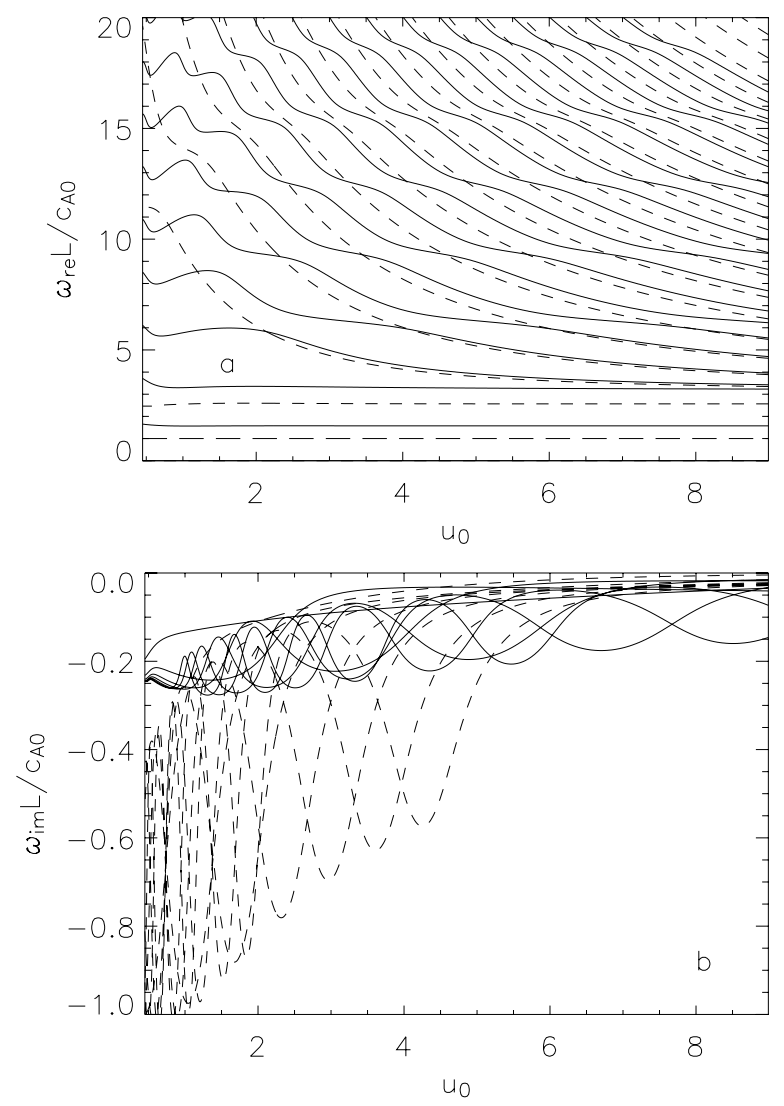

Fig. 6. Logarithmic plots of the a) real and b) imaginary parts of $\omega L / c_{\mathrm{Ac}}$ against the parameter $u_{0}$ for vertical modes. The density ratio has been fixed as $\rho_{\mathrm{l}} / \rho_{\mathrm{c}}=10$ and the width parameter as $a=0.5$ (solid lines) and $a=0.1$ (dashed lines). Notice that $u_{0} \geq a$ is required, so the curves can not reach small values of $u_{0}$. Only the 20 lowest values in panel a) and the 7 lowest values in panel b) have been represented for each family.

cavity modes away from the avoided crossings. An interesting feature is that the imaginary part of the frequency is increased as $u_{0}$ is decreased, signalling that more eccentric arcades have higher damping times.

As it was discussed in Sect. 2, the limit of a circular arcade (and the straight slab) can be obtained if we allow the parameter $u_{0}$ to be large, or if the eccentricity in Eq. (5) is small. Figure 7 shows how this comparison is achieved by keeping constant the arcade width in the summit compared with its length, $D_{2} /(2 L)=a_{\text {cart }} / L$, with these two quantities defined in Eqs. (10) and (11). Therefore, the parameter $a$ in the elliptical arcade depends on $u_{0}$ as

$a=\operatorname{arcsinh}\left[2 E(\epsilon, \pi / 2) \frac{D_{2}}{2 L}\right]$.

Notice in Fig. 7 that as the eccentricity of the arcade tends to zero $\left(u_{0} \gg 1\right)$ the avoided crossings follow a line. This line is similar to the frequency of the modes of a Cartesian straight model (and also a cylindrical model), as can be seen in the panel. However, the elliptical model has also the cavity modes, not present in the other geometries. It is very difficult to follow the avoided crossings for investigating the dependence of the arcade modes on the eccentricity, but the trend is that as the eccentricity is increased the frequency of these modes is increased with respect to the modes of the cylindrical model and even surpass the frequency of the modes of the straight model. For $\varepsilon \approx 1$ the arcade modes in the elliptical geometry do not tend to the modes of the straight slab and the periods for the elliptical geometry 


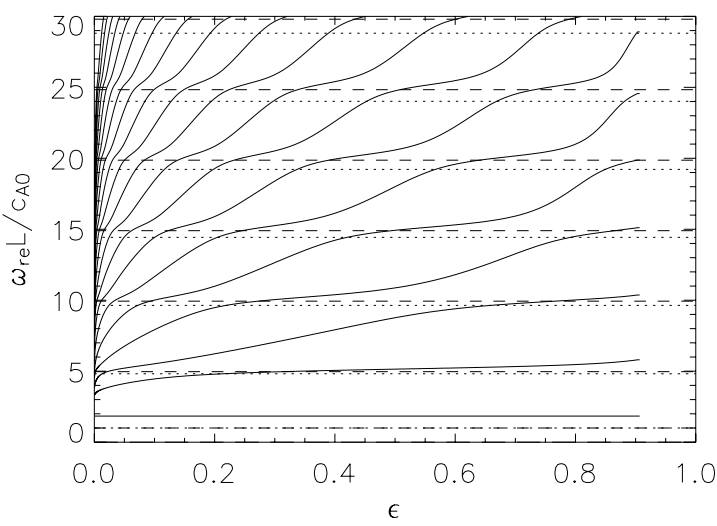

Fig. 7. Plots of the real part of $\omega L / c_{\mathrm{Ac}}$ against the eccentricity $\varepsilon$ for vertical modes $(m=1)$. The density ratio has been fixed as $\rho_{\mathrm{l}} / \rho_{\mathrm{c}}=10$ and the width of the arcade as $D_{2} /(2 L)=0.1$, with the $a$ parameter calculated with Eqs. (48). The frequencies of the straight and cylindrical arcade models are overplotted as horizontal dashed lines and dotted lines, respectively. Only the 20 lowest values for the elliptical geometry have been represented.

are slightly lower than those of the Cartesian slab, although in this limit the arcade is almost lying flat and close to be straight, but even in this limit the footpoints are connecting the horizontal photosphere instead of two vertical ones at $x= \pm L$ in the Cartesian model. Nevertheless, the avoided crossings with the cavity modes (which are not present in the cylindrical and Cartesian geometries) make difficult to perform numerical comparisons, and in any case the frequency shifts are very small.

Next, the dependence of the frequencies on the equilibrium density contrast between the arcade and the coronal medium $\left(\rho_{1} / \rho_{\mathrm{c}}\right)$ is considered in Fig. 8. The agreement of the frequencies with the approximate formulae deduced for the elliptical cavity (Eq. (43)) and the elliptical ring (Eq. (46)) is remarkable, and when these curves cross each other the exact solution presents an avoided crossing, particularly in the limit of high density contrast. In Fig. $8 \mathrm{~b}$ it can be checked that the confinement is better for arcades with high density contrast (the imaginary part tends to zero as $\rho_{1} / \rho_{\mathrm{c}} \rightarrow \infty$ ). All the imaginary parts of the modes tend to infinity as $\rho_{\mathrm{l}} / \rho_{\mathrm{c}} \rightarrow 0$, since no modes are allowed in a semi infinite homogeneous plane.

Finally, the results of the elliptical model are compared with those of the straight and cylindrical models (Fig. 9). We check in panel a (real part of the frequency) that the cavity modes do not have any counterparts in this geometry (although they appear in the cylindrical model for other equilibrium profiles), as it has been commented early in this section. For thin arcades the three geometries give similar values of $\omega L / c_{\mathrm{Ac}}$ for the arcade modes, and hence, similar periods. However, as the thickness is increased the cylindrical geometry has no longer the same periods, but the elliptical and the straight one are still in a good agreement. This is because the elliptical arcade is still far from filling the cavity with dense material, while in the cylindrical model the region of low density plasma below the arcade is becoming small. The plot is similar if other values of the eccentricity are considered, except that the real part of the frequency for the cavity modes is modified (Fig. 6a) and the imaginary part of the elliptical modes is increased as $u_{0}$ is lowered (Fig. 6b).

On the other hand, Fig. 9b (imaginary part of the frequency) shows a very important conclusion: the damping due to the coronal leakage depends highly on the geometry. As noted in Díaz et al. (2006), the cylindrical model has a damping time roughly two times the one in the straight Cartesian slab. On the other
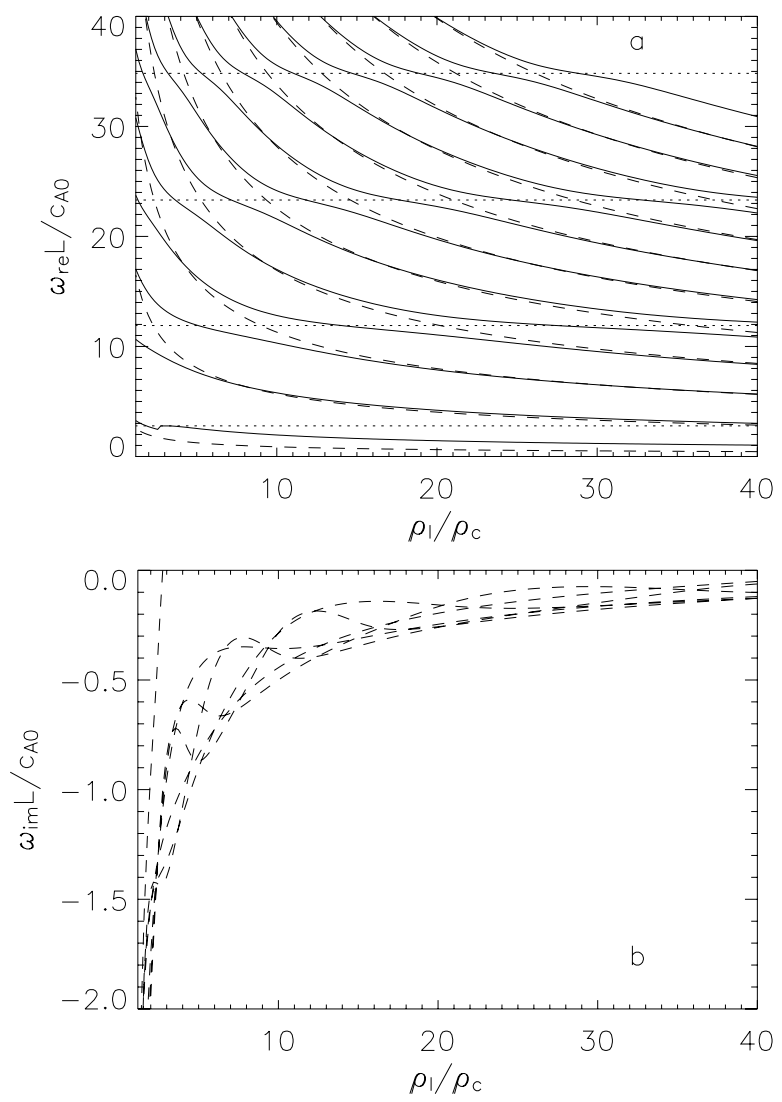

Fig. 8. Plots of the a) real part and b) imaginary part of $\omega L / c_{\mathrm{Ac}}$ against the density contrast $\rho_{\mathrm{l}} / \rho_{\mathrm{c}}$ for vertical modes $(m=1)$. The parameters have been fixed as $u_{0}=1$ and $D_{2} /(2 L)=0.1$ (so $a=0.245$ ). In panel a) the curves of a elliptical ring (Eq. (46)) have been overplotted as dashed lines and the curves of the elliptical cavity (Eq. (43)) have been overplotted in dotted lines. Only the 14 lowest values for the elliptical geometry have been represented in panel a) and the 7 lowest in panel b).

hand, the elliptical model gives an even longer damping rates for the arcade modes (lower part of the band of frequencies), so the coronal leakage is not efficient in this model to explain the observed damping times for coronal structures such as loops.

\subsection{Spatial structure}

The solutions represented by Eq. (33) are two-dimensional, with non-trivial dependence on the elliptical coordinates $(u, v)$. However, in this equilibrium the main different part is the dependence on $u$ via $f(u)$, so we concentrate on this plots first.

The spatial structure of the first seven eigenfunctions is represented in Fig. 10 for a typical set of parameters. In this range there is one single trapped mode, which decays exponentially as $u \rightarrow \infty$. The rest of the modes are leaky and propagate towards $u \rightarrow \infty$, and since we are considering stationary solutions they grow exponentially as the distance from the arcade is increased (in a similar way to previous works, see Cally 1986, 2003; Stenuit et al. 1998; Díaz et al. 2004). The solutions are continuous and smooth at the boundaries of the arcade, as the boundary conditions demand.

The distinction between kink and sausage modes is not evident in these problems, and the modes can be difficult to assign to such categories. For example, in Fig. 10 the first, fifth and sixth are kink modes (both boundaries oscillating in phase), while the second, third and seventh are sausage (both boundaries 

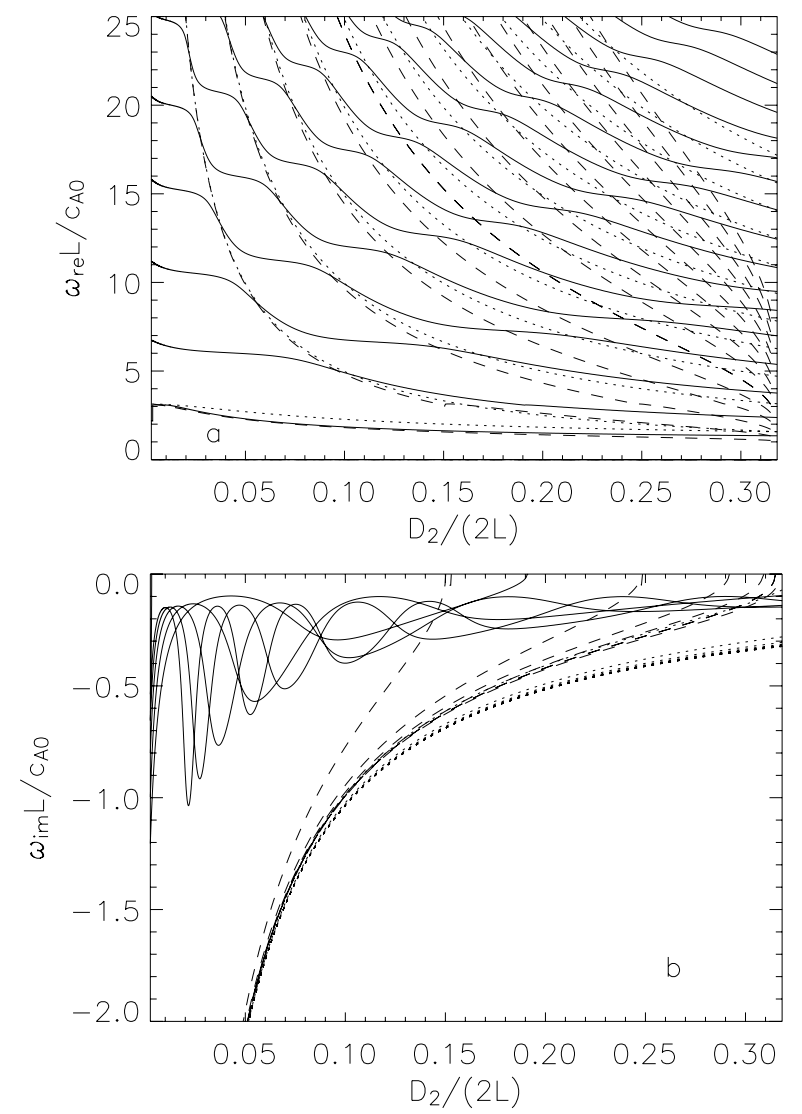

Fig. 9. Logarithmic plots of the a) real and b) imaginary parts of $\omega L / c_{\mathrm{Ac}}$ against the arcade half-width for vertical modes $(m=1)$. The density ratio has been fixed as $\rho_{\mathrm{l}} / \rho_{\mathrm{c}}=10$ and the eccentricity parameter as $u_{0}=2$. The results of the elliptical model are plotted in solid lines, while the frequencies for the cylindrical and straight models are plotted in dashed and dotted lines, respectively. Only the 15 lowest values in each geometry have been represented ( 5 for the elliptical model in panel b).

oscillating in opposition of phase). Notice that sausage modes have a displacement of the centre of the slab. It is also interesting to see that some modes have mixed characteristics, like the forth mode, which again points out that this distinction is no longer inherent to the problem.

We can also see in Fig. 10 the different spatial properties of arcade modes (first,third and fifth modes for these parameters) compared with the cavity modes: the amplitude below the arcade $\left(0 \leq u<u_{0}-a\right)$ is relatively smaller. Notice that the order of the mode is also the number of extrema that it displays between $0 \leq u<u_{0}+a$. There is no relation between the order of the mode and its character; for example, the sixth and seventh modes are both cavity modes, but they can have different properties for other choices of the parameters.

Finally, we can combine this dependence with the dependence along the magnetic field (the $v$ direction), which leads to vertical and swaying modes, as in Díaz et al. (2006). It is important to remark that despite the equilibrium magnetic field being singular at the foci of the ellipses, the solution is regular in all the domain. In Fig. 11 the velocity vector field at $t=0$ is represented with its dependence in the two dimensions, superimposed with the initial position of the arcade. Three harmonics have been chosen to reflect the three types of modes: the trapped ones ( $n=1$ for this choice of parameters), the leaky ones with amplitude mainly in the cavity $(n=2)$ and the leaky ones with amplitude in the arcade $(n=3)$, although we see that there is an amplitude of a similar value in the cavity for these last type of mode, but since the plasma in the arcade is denser the energy is mainly in the arcade itself.

As in the cylindrical model, the two families of modes have a different behaviour. The vertical modes $(m=1)$ have displacement of the arcade mainly on the $x$-direction (apparent, since all the motions are in the $u$-direction), but the swaying modes $(m=2)$ displace apparently the arcade in the direction along the photosphere, and distort the shape quite more notably that in the cylindrical geometry. However, this distortion is only apparent, since all the movements are in the radial direction, but depending on the point of view of an observer, these swaying modes could be difficult to identify as fast MHD modes in observations. It cannot be appreciated in the plots presented here, but the amplitude is better confined in the arcade for the trapped swaying (and higher order) modes that for the trapped vertical mode, in accordance with the results for the Cartesian slab.

\section{Conclusions}

The linearised MHD wave equations have been written for an equilibrium configuration described in elliptical cylindrical coordinates, with the magnetic field and density varying in the direction across the arcade. The resulting differential equations have been solved analytically in the low-beta limit using separation of variables, and a two-dimensional solution has been obtained.

First of all, the results obtained in Cartesian and cylindrical geometries are very close to the modes of the elliptical geometry. The first major difference is that the elliptical arcade supports another type of modes: those having amplitude mainly in the coronal region below the arcade. Obviously, these modes cannot be described in the Cartesian geometry, and in the cylindrical geometry they are not present for this choice of density profile in the radial direction, since in the cylindrical model the Alfvén speed tends to zero as $r \rightarrow 0$ (Verwichte et al. 2006a, Fig. 2). On the other hand, the elliptical configuration has a region with the Alfvén speed different to zero near the origin and it is only vanishing in the foci (Fig. 3b), and hence modes can be trapped in this zone. It has also been checked that these modes have a similar frequency to the ones of an elliptical oscillatory membrane (Eq. (43)) and that their amplitude is mainly below the arcade (Fig. 11). Notice that these modes are present in other cylindrical equilibrium density profiles (such as in Díaz et al. 2006). However, these modes are difficult to observe in solar coronal arcades, since the amplitude in the dense (visible) part is small. Moreover, structures such as coronal loops are better described in terms of toroidal coordinates, and in such systems there is no cavity below the dense structure where this type of mode can be trapped. That is the reason why this model is better suited for coronal arcades, since a loop needs to be described using the three dimensions, although some of the results found can be extrapolated to coronal loop oscillations.

However, compared with a loop with elliptical cross-section, the elliptical arcade does not have two families that become degenerated in the limit of low eccentricity (Ruderman 2003). This is because our line-tying boundary conditions limit freedom to oscillate in different directions. A loop whose height is larger that its footpoint half-separation could be studied by applying the line-tying in the plane $y=0$ and restricting $v$ to the range $[-\pi / 2, \pi / 2]$, but that seems to be less common in the solar corona (and the arcade modelled in this geometry would be thinner in the apex that in the footpoints). Notice also that there are no unstable modes in this problem and the equilibrium structure 

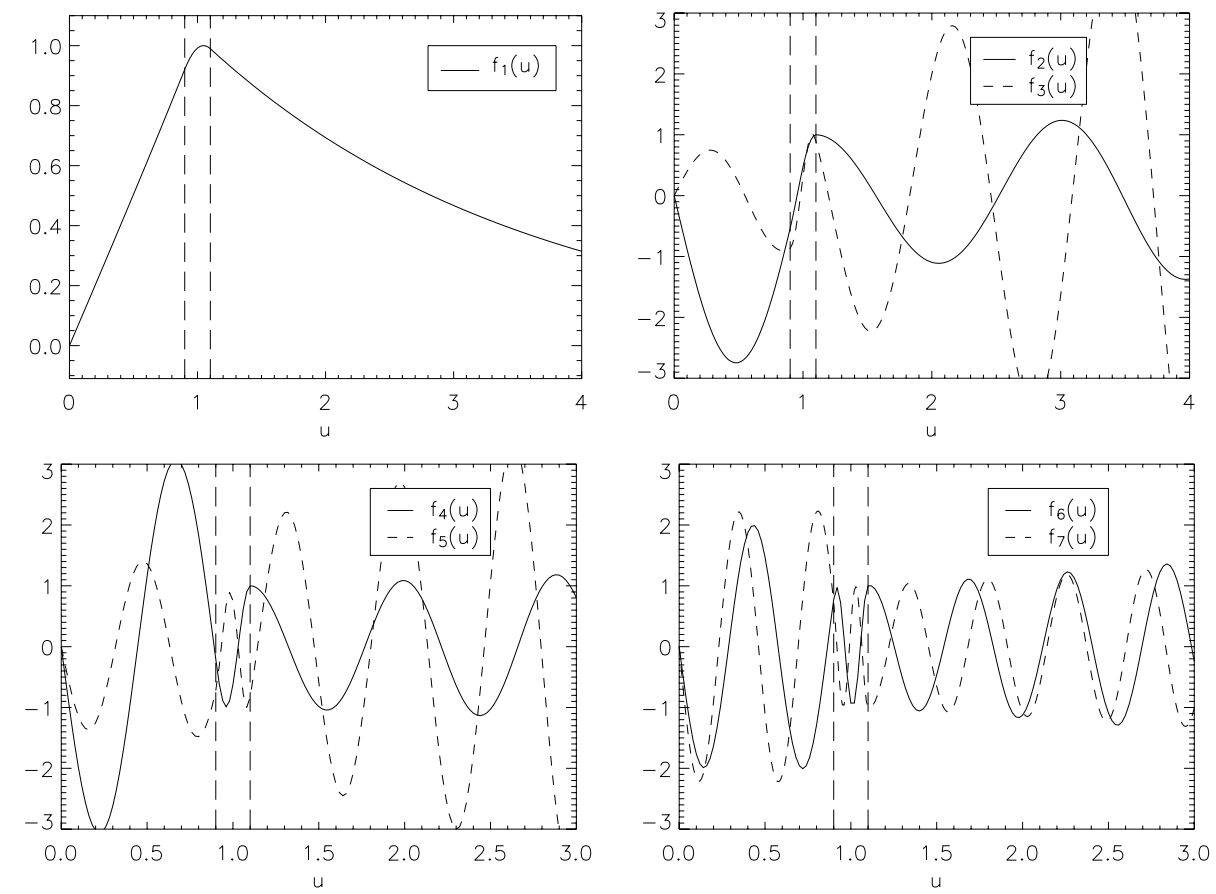

Fig. 10. Panels showing the spatial structure of the $u$-dependent part of the perturbed velocity $f_{n}(u)$ (first seven vertical harmonics, $m=1$ ) for $\rho_{\mathrm{l}} / \rho_{\mathrm{c}}=10, u_{0}=1$ and $a=0.1$. All the functions have been normalised so the highest value of the amplitude in the dense region is equal to 1 . The limits of the region with dense plasma have been marked with vertical dashed lines.
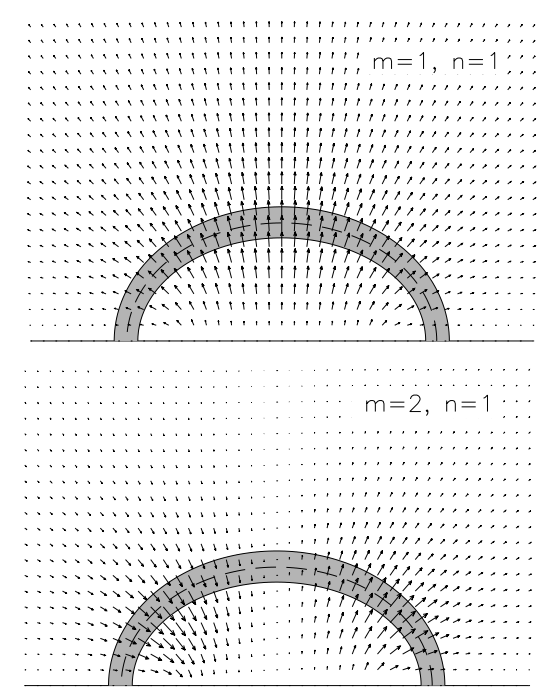

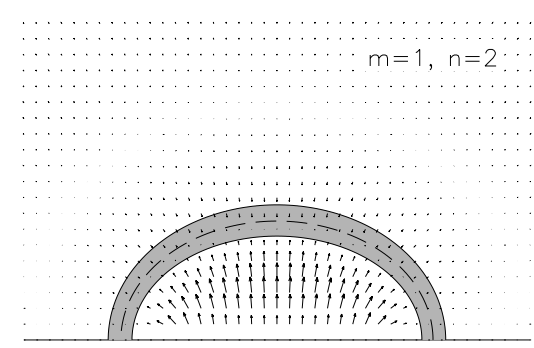

$m=2, n=2$

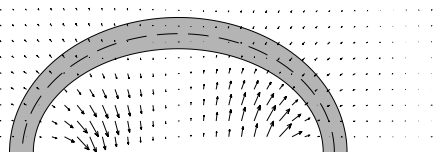

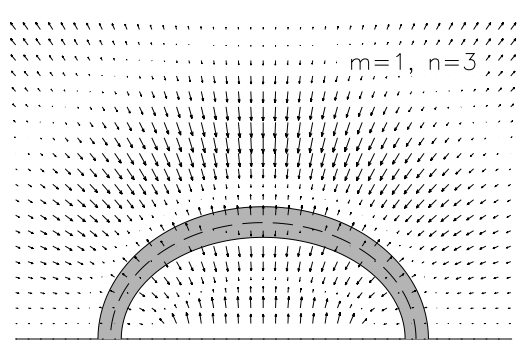

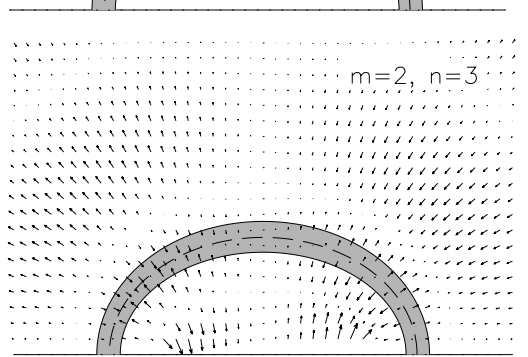

Fig. 11. Vector field plot of the perturbed velocity (Eq. (22)) at $t=0$ in the $x y$-plane. The mode family (vertical $m=1$ and swaying $m=2$ ) and the order of the harmonic has been included in the legend. The initial position of the arcade is overplotted in each panel as a grey region.

is not collapsing because the magnetic tension is opposed by the magnetic pressure.

As in the cylindrical geometry, the different harmonics of the Cartesian slab in the direction along the field lines are no longer multiples and become different families as $m$ is changed (vertical and swaying modes). In a three-dimensional object there would be an extra type of family: those oscillating in the $z$-direction of our model. We can see in Fig. 12 the perturbations from a privileged point of view (the arcade is seen head on form the side), but in the context of solar observations a perturbation such as the swaying one would seem to have a component along the field lines and could be mistakenly identified as a slow mode. On the other hand, the distinction between kink and sausage modes is not relevant for this geometry.
One of the main aims of this work is to test the effect of curvature in the modes of a straight Cartesian slab (Edwin \& Roberts 1982). In that sense, the results of the elliptical geometry confirm those of the cylindrical geometry (Verwichte et al. 2006a), since the periods we obtain are very close to those of the straight slab. However, the damping times are remarkably different, and show that coronal leakage seems to be insufficient to explain the damping in some coronal observations. The elliptical model has some ingredients that are not present in the straight slab, such as having a single photospheric surface, a larger thickness in the apex compared with that in the footpoints and a curvature that depends on the position. Despite these aspects, our model confirms that the straight model gives a quite accurate estimation of the modes and is easier to work with. It is 


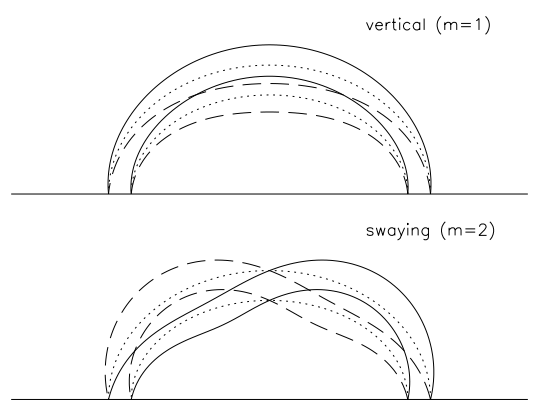

Fig. 12. Plots of the boundaries of an arcade oscillating with the fundamental kink vertical and swaying modes. The boundaries have been plotted for different instants during a period $P: t / P=0$ (initial position), $t / P=1 / 4$ and $t / P=3 / 4$ in dotted, solid and dashed lines, respectively. The density ratio has been fixed as $\rho_{1} / \rho_{\mathrm{c}}=10$, the eccentricity parameter as $u_{0}=1$ and the width parameter as $a=0.1$. The amplitude has been chosen to show better the displacements, but in reality it should be smaller to be adequately described by the linear regime.

remarkable that even the fundamental mode becomes leaky for certain ranges of the parameters.

Regarding the different families of MHD modes, it has been shown in our analysis that they are decoupled (see Eqs. (22) and (24)). However, previous works have shown that many effects contribute to couple these modes in the corona, especially when gravitational stratification and curvature of field lines is included (Beliën et al. 1997; Arregui et al. 2001, 2003, 2004a,b; Bogdan et al. 2003; Hasan et al. 2005). Here, structure is caused by the divergence of the field lines. Thus, the expansion of the field lines alone does not induce coupling in our model unless some sort of dependence on the z-coordinate is allowed in Eq. (20). Further work is expected to investigate the role of the continous Alfvén spectrum and its coupling with the discrete fast modes.

An important unanswered question is whether these conclusions are modified when other equilibrium density profiles are chosen instead of Eq. (8). In cylindrical geometry there are some changes in the damping rate and the cavity modes (Díaz et al. 2006; Verwichte et al. 2006a), but it remains an open question to check those results in the elliptical geometry. It would be also interesting to compare the cavity modes that also are present in the cylindrical geometry for different profiles. Another assumption that needs to be explored further is the effect of a longitudinal propagation in the $z$-direction. Including this term couples the Alfvén and fast modes by coupling their equations, and could lead to damping due to resonant absorption.

On the other hand, the equilibrium presented in this paper can be extended to other problems, since it is still simple enough to be solved analytically and the parameter space is small, but has other effects not previously taken into account, such as divergence of the field lines and the possibility to explore the role of the eccentricity. Further comparisons with observational data can also be taken, in particular for the damping rates, since the shift in the periods of the fast modes has been proved to be relatively small.

Acknowledgements. Economic support from PPARC on the St Andrews Solar Theory Rolling Grant is acknowledged. The author also thanks Prof. B. Roberts for discussion and comments.

\section{References}

Abramowitz, M., \& Stegun, I. A. 1967, Handbook of Mathematical Functions (Dover)

Andries, J., Goossens, M., \& Arregui, I. 2005, ApJ, 624, L57

Andries, J., Goossens, M., Hollweg, J. V., Arregui, I., \& Van Doorsselaere, T. 2005, A\&A, 430, 1109

Arregui, I., Oliver, R., \& Ballester, J. L. 2001, A\&A, 369, 1122

Arregui, I., Oliver, R., \& Ballester, J. L. 2003, A\&A, 402, 1129

Arregui, I., Oliver, R., \& Ballester, J. L. 2004a, ApJ, 602, 1020

Arregui, I., Oliver, R., \& Ballester, J. L. 2004b, A\&A, 425, 729

Aschwanden, M. J. 2004, Physics of the corona, Springer Praxis Books in Geophysical Sciences

Beliën, A. J. C., Poedts, S., \& Goedbloed, J. P. 1997, A\&A, 322, 995

Bogdan, T. J., Carlsson, M., Hansteen, V. H., et al. 2003, ApJ, 599, 626

Brady, C. S., \& Arber, T. D. 2005, A\&A, 438, 733

Cally, P. S. 1986, Sol. Phys., 103, 277

Cally, P. S. 2003, Sol. Phys., 217, 95

Díaz, A. J. 2004, Ph.D. Thesis, Universitat de les Illes Balears

Díaz, A. J., Oliver, R., Ballester, J. L., \& Roberts, B. 2004, A\&A, 424, 1055

Díaz, A. J., Zaqarashvili, T., \& Roberts, B. 2006, A\&A, 455, 709

Donnelly, G.R., Díaz, A. J., \& Roberts, B. 2006, A\&A, in press

Edwin, P. M., \& Roberts, B. 1982, Sol. Phys., 76, 239

Edwin, P. M., \& Roberts, B. 1983, Sol. Phys., 88, 179

Goedbloed, J. P. 1983, Lecture Notes on Ideal Magnetohydrodynamics, Rijnhuizen Report

Hasan, S. S., van Ballegooijen, A. A., Kalkofen, W., \& Steiner, O. 2005, ApJ, 631,1270

McEwan, M., Donnelly, G. R., Díaz, A. J., \& Roberts, B. 2006, A\&A, accepted Nakariakov, V. N., \& Verwichte, E. 2005, Living Rev. Solar Phys., 2, http: //www . livingreviews . org/lrsp-2005-3

Oliver, R., Ballester, J. L., Hood, A. W., \& Priest, E. R. 1993, A\&A, 273, 647

Oliver, R., Hood, A. W., \& Priest, E. R. 1996, ApJ, 461, 424

Roberts, B., Edwin, P. M., \& Benz, A. O. 1984, ApJ, 279, 857

Ruderman, M. S. 2003, A\&A, 409, 287

Selwa, M., Murawski, K., Solanki, S. K., Wang, T. J., \& Tóth, G. 2005, A\&A, 440,385

Smith, J. M., Roberts, B., \& Oliver, R. 1997, A\&A, 317, 752

Spiegel, M. R. 1968, Mathematical Handbook of Formulas and Tables (McGraw-Hill)

Stenuit, H., Keppens, R., \& Goossens, M. 1998, A\&A, 331, 392

Terradas, J., Oliver, R., \& Ballester, J. L. 2005, A\&A, 441, 371

Van Doorsselaere, T., Debosscher, A., Andries, J., \& Poedts, S. 2004, A\&A, 424, 1065

Verwichte, E., Nakariakov, V. M., Ofman, L., \& Deluca, E. E. 2004, Sol. Phys., 223, 77

Verwichte, E., Nakariakov, V. M., \& Cooper, F. C. 2005, A\&A, 430, L65

Verwichte, E., Foullon, C., \& Nakariakov, V. M. 2006a, A\&A, 446, 1139

Verwichte, E., Foullon, C., \& Nakariakov, V. M. 2006b, A\&A, 449, 769 